\title{
Exact Solutions to the Generalized Benjamin Equation
}

\author{
Hua Gao, Genhu Di \\ Department of Applied Mathematics, Yuncheng University, Yuncheng, China \\ Email: gaohuaxx@126.com
}

Received 26 March 2014; revised 26 April 2014; accepted 3 May 2014

Copyright (C) 2014 by authors and Scientific Research Publishing Inc.

This work is licensed under the Creative Commons Attribution International License (CC BY). http://creativecommons.org/licenses/by/4.0/

(c) (i) Open Access

\section{Abstract}

Based on the $\left(\frac{G^{\prime}}{G}\right)$-expansion method, a series of exact solutions of the generalized Benjamin equation have been obtained. The travelling wave solutions are expressed by the hyperbolic functions, the trigonometric functions and the rational functions. It is shown that the $\left(\frac{G^{\prime}}{G}\right)$-expansion method is concise, and its applications are promising.

Keywords

$\left(\frac{G^{\prime}}{G}\right)$-Expansion Method, Generalized Benjamin Equation, Nonlinear Evolution Equations

\section{Introduction}

It is well known that seeking exact solutions for nonlinear evolution equations (NLEES) plays an important role in mathematical physics. In the past five decades or so, many effective methods have been presented, which contain the inverse scattering method [1], Hirota bilinear method [2], the tanh-function method and its various extension [3] [4], the sine-cosine function method [5] [6], homogeneous balance method [7] [8], Jacobi elliptic function method [9] [10], the first-integral method [11] [12], the sine-Gordon equation method [13] and the Exp-function method [14]-[16].

Recently, Wang et al. [17] proposed a new method called the $\left(\frac{G^{\prime}}{G}\right)$-expansion method to look for travelling wave solutions of nonlinear evolution equations (NLEEs). The $\left(\frac{G^{\prime}}{G}\right)$-expansion method is based on the assumptions that the travelling wave solutions can be expressed by a polynomial in $\left(\frac{G^{\prime}}{G}\right)$ as follows: 


$$
u=\sum_{i=0}^{m} a_{i}\left(\frac{G^{\prime}}{G}\right)^{i}
$$

where $G=G(\eta)$ satisfies a second order linear ordinary differential equation (LODE):

$$
G^{\prime \prime}+\lambda G^{\prime}+\mu G=0
$$

where $G^{\prime}=\frac{\mathrm{d} G(\eta)}{\mathrm{d} \eta}, G^{\prime \prime}=\frac{\mathrm{d}^{2} G(\eta)}{\mathrm{d} \eta^{2}}, \eta=x-V t$, and $V$ is a constant. The degree of the polynomial can be determined by considering the homogeneous balance between the highest order derivative and nonlinear terms appearing in the given NLEE. The coefficients of the polynomial can be obtained by solving a set of algebraic equations resulted from the process of using the method. By using the $\left(\frac{G^{\prime}}{G}\right)$-expansion method, many nonlinear equations [17]-[27] have been successfully solved.

In the present paper, we will extend the $\left(\frac{G^{\prime}}{G}\right)$-expansion method to the following generalized Benjamin equation:

$$
u_{t t}+\alpha\left(u^{n} u_{x}\right)_{x}+\beta u_{x x x x}=0
$$

where $\alpha$ and $\beta$ are constants. Equation (3) is used in the analysis of long wave in shallow water; see [28]. To the best of our knowledge, there are a few articles about this equation. Recently, by applying the extended tanh method, Taghizadeh et al. [29] obtained some exact solutions. In the subsequent section, we will illustrate the $\left(\frac{G^{\prime}}{G}\right)$-expansion method in detail with the generalized Benjamin equation.

\section{Exact Solutions to the Generalized Benjamin Equation}

First, in this section, we start out our study for Equation (3). Firstly, making the following wave variable

$$
u(x, t)=U(\eta), \eta=k x+w t,
$$

where $k$, and $w$ are constants to be determined later, Equation (3) becomes the ODE

$$
w^{2} U^{\prime \prime}+\alpha k^{2}\left(U^{n} U^{\prime}\right)^{\prime}+\beta k^{4} U^{\prime \prime \prime \prime}=0,
$$

where the prime denotes the derivation with respect to $\eta$. Integrating Equation (5), twice and setting the constant of integrating to zero, we obtain

$$
w^{2} U+\frac{\alpha k^{2}}{n+1} U^{n+1}+\beta k^{4} U^{\prime \prime}=0
$$

Then, making the following transformation

$$
\varphi=U^{n}
$$

We can obtain an equation for $\varphi$ as

$$
w^{2} n^{2}(n+1) \varphi^{2}+\alpha k^{2} n^{2} \varphi^{3}+\beta k^{4}\left[n(n+1) \varphi \varphi^{\prime \prime}-\left(n^{2}-1\right) \varphi^{\prime 2}\right]=0 .
$$

Now, we make an ansatz (1) for the solution of Equation (8). Balancing the terms $\varphi^{3}$ and $\varphi \varphi^{\prime \prime}$ in Equation (8) yields the leading order $m=2$. Therefore, we can write the solution of Equation (8) in the form

$$
\varphi(\eta)=a_{2}\left(\frac{G^{\prime}}{G}\right)^{2}+a_{1}\left(\frac{G^{\prime}}{G}\right)+a_{0}
$$

Substituting (2) and (9) into (8), collecting the coefficients of $\left(\frac{G^{\prime}}{G}\right)^{i} \quad(i=0, \cdots, 6)$ and set it to zero we ob- 
tain the following system of algebraic equations for: $a_{0}, a_{1}, a_{2}, k, w, \lambda$ and $\mu$ :

$$
\begin{aligned}
& \left(\frac{G^{\prime}}{G}\right)^{6}: 2 \beta k^{4} n^{2} a_{2}^{2}+6 \beta k^{4} n a_{2}^{2}+4 \beta k^{4} a_{2}^{2}+\alpha k^{2} n^{2} a_{2}^{3}=0, \\
& \left(\frac{G^{\prime}}{G}\right)^{5}: 4 \beta k^{4} a_{2} a_{1}+10 \beta k^{4} n a_{2}^{2} \lambda+8 \beta k^{4} a_{2}^{2} \lambda+2 \beta k^{4} n^{2} a_{2}^{2} \lambda+3 \alpha k^{2} n^{2} a_{1} a_{2}^{2}+8 \beta k^{4} n a_{2} a_{1}+4 \beta k^{4} n^{2} a_{2} a_{1}=0, \\
& \left(\frac{G^{\prime}}{G}\right)^{4}: 4 \beta k^{4} n a_{2}^{2} \lambda^{2}+3 \alpha k^{2} n^{2} a_{2} a_{1}^{2}+\beta k^{4} a_{1}^{2}+4 \beta k^{4} a_{2}^{2} \lambda^{2}+2 \beta k^{4} n a_{1}^{2}+\beta k^{4} n^{2} a_{1}^{2}+w^{2} n^{3} a_{2}^{2}+6 \beta k^{4} n^{2} a_{2} a_{0}
\end{aligned}
$$
$+3 \alpha k^{2} n^{2} a_{2}^{2} a_{0}+8 \beta k^{4} a_{2} a_{1} \lambda+13 \beta k^{4} n a_{2} a_{1} \lambda+w^{2} n^{2} a_{2}^{2}+5 \beta k^{4} n^{2} a_{2} a_{1} \lambda+6 \beta k^{4} n a_{2} a_{0}+8 \beta k^{4} a_{2}^{2} \mu+8 \beta k^{4} n a_{2}^{2} \mu=0$, $\left(\frac{G^{\prime}}{G}\right)^{3}: 2 \beta k^{4} a_{1}^{2} \lambda+10 \beta k^{4} n^{2} a_{2} a_{0} \lambda+6 \alpha k^{2} n^{2} a_{2} a_{1} a_{0}+3 \beta k^{4} n a_{1}^{2} \lambda-2 \beta k^{4} n^{2} a_{2}^{2} \lambda \mu+2 \beta k^{4} n^{2} a_{1} a_{0}+8 \beta k^{4} a_{2}^{2} \lambda \mu$ $+2 w^{2} n^{2} a_{2} a_{1}+2 w^{2} n^{3} a_{2} a_{1}+8 \beta k^{4} a_{2} a_{1} \mu+10 \beta k^{4} n a_{2} a_{1} \mu+10 \beta k^{4} n a_{2} a_{0} \lambda+\beta k^{4} n^{2} a_{2} a_{1} \lambda^{2}+4 \beta k^{4} a_{2} a_{1} \lambda^{2}$ $+\beta k^{4} n^{2} a_{1}^{2} \lambda+2 \beta k^{4} n a_{1} a_{0}+5 \beta k^{4} n a_{2} a_{1} \lambda^{2}+\alpha k^{2} n^{2} a_{1}^{3}+6 \beta k^{4} n a_{2}^{2} \lambda \mu+2 \beta k^{4} n^{2} a_{2} a_{1} \mu=0$, $\left(\frac{G^{\prime}}{G}\right)^{2}:-\beta k^{4} n^{2} a_{2} a_{1} \lambda \mu+3 \alpha k^{2} n^{2} a_{2} a_{0}^{2}+8 \beta k^{4} n a_{2} a_{0} \mu+w^{2} n^{3} a_{1}^{2}+2 \beta k^{4} n a_{1}^{2} \mu+3 \alpha k^{2} n^{2} a_{1}^{2} a_{0}+4 \beta k^{4} n a_{0} a_{2} \lambda^{2}$ $+7 \beta k^{4} n a_{2} a_{1} \lambda \mu+2 \beta k^{4} a_{1}^{2} \mu+8 \beta k^{4} a_{2} a_{1} \lambda \mu+2 n^{2} a_{0} a_{2} w^{2}+3 \beta k^{4} n a_{1} a_{0} \lambda-2 \beta k^{4} n^{2} a_{2}^{2} \mu^{2}+\beta k^{4} n a_{1}^{2} \lambda^{2}$ $+8 \beta k^{4} n^{2} a_{2} a_{0} \mu+2 \beta k^{4} n a_{2}^{2} \mu^{2}+4 \beta k^{4} a_{2}^{2} \mu^{2}+4 \beta k^{4} n^{2} a_{0} a_{2} \lambda^{2}+2 w^{2} n^{3} a_{2} a_{0}+3 \beta k^{4} n^{2} a_{1} a_{0} \lambda+\beta k^{4} a_{1}^{2} \lambda^{2}$ $+n^{2} a_{1}^{2} w^{2}=0$, $\left(\frac{G^{\prime}}{G}\right)^{1}: \beta k^{4} n a_{1}^{2} \lambda \mu+6 \beta k^{4} n a_{0} a_{2} \mu \lambda+4 \beta k^{4} a_{1} a_{2} \mu^{2}+2 \beta k^{4} a_{1}^{2} \lambda \mu+2 \beta k^{4} n^{2} a_{1} a_{0} \mu-\beta k^{4} n^{2} a_{1}^{2} \lambda \mu+2 \beta k^{4} n a_{1} a_{2} \mu^{2}$ $+2 w^{2} n^{3} a_{1} a_{0}+\beta k^{4} n^{2} a_{0} a_{1} \lambda^{2}+3 \alpha k^{2} n^{2} a_{0}^{2} a_{1}-2 \beta k^{4} n^{2} a_{1} a_{2} \mu^{2}+6 \beta k^{4} n^{2} a_{0} a_{2} \mu \lambda+\beta k^{4} n a_{0} a_{1} \lambda^{2}+2 \beta k^{4} n a_{1} a_{0} \mu+2 w^{2} n^{2} a_{1} a_{0}=0$, $\left(\frac{G^{\prime}}{G}\right)^{0}: w^{2} n^{3} a_{0}^{2}+w^{2} n^{2} a_{0}^{2}+\alpha k^{2} n^{2} a_{0}^{3}+\beta k^{4} a_{1}^{2} \mu^{2}+\beta k^{4} n^{2} a_{0} a_{1} \lambda \mu-\beta k^{4} n^{2} a_{1}^{2} \mu^{2}+2 \beta k^{4} n a_{0} a_{2} \mu^{2}+2 \beta k^{4} n^{2} a_{0} a_{2} \mu^{2}$ $+\beta k^{4} n a_{0} a_{1} \lambda \mu=0$.

Solving the above system by Matlab gives

$$
\begin{gathered}
a_{0}=-\frac{2 \beta k^{2} \mu\left(n^{2}+3 n+2\right)}{\alpha n^{2}}, a_{1}=-\frac{2 \beta k^{2} \lambda\left(n^{2}+3 n+2\right)}{\alpha n^{2}}, \\
a_{2}=-\frac{2 \beta k^{2}\left(n^{2}+3 n+2\right)}{\alpha n^{2}}, w= \pm \frac{\sqrt{4 \beta \mu-\lambda^{2} \beta}}{n},
\end{gathered}
$$

where $\lambda, k$, and $\mu$ are arbitrary constants.

Substituting (10) (11) into (9) yields:

$$
\varphi(\eta)=-\frac{2 \beta k^{2}\left(n^{2}+3 n+2\right)}{\alpha n^{2}}\left(\frac{G^{\prime}}{G}\right)^{2}-\frac{2 \beta k^{2} \lambda\left(n^{2}+3 n+2\right)}{\alpha n^{2}}\left(\frac{G^{\prime}}{G}\right)-\frac{2 \beta k^{2} \mu\left(n^{2}+3 n+2\right)}{\alpha n^{2}},
$$

where $\eta=k x \pm \frac{\sqrt{4 \beta \mu-\lambda^{2} \beta}}{n} t$.

Substituting the general solutions of Equation (2) into the formulae (12) we have three types of travelling wave solutions of the generalized Benjamin equation as follows:

When $\lambda^{2}-4 \mu>0$,

$$
u_{1}=\left[-\gamma\left(\frac{C_{1} \sinh \frac{1}{2} \sqrt{\lambda^{2}-4 \mu} \eta+C_{2} \cosh \frac{1}{2} \sqrt{\lambda^{2}-4 \mu} \eta}{C_{1} \cosh \frac{1}{2} \sqrt{\lambda^{2}-4 \mu} \eta+C_{2} \sinh \frac{1}{2} \sqrt{\lambda^{2}-4 \mu} \eta}\right)^{2}+\gamma\right]
$$


where $\eta=k x \pm \frac{\sqrt{4 \beta \mu-\lambda^{2} \beta}}{n} t, \gamma=\frac{\beta k^{2}\left(n^{2}+3 n+2\right)\left(\lambda^{2}-4 \mu\right)}{2 \alpha n^{2}}, C_{1}$ and $C_{2}$ are arbitrary constants. It is easy to see that the hyperbolic solution (13) can be rewritten at $C_{1}^{2}>C_{2}^{2}$, as follows

$$
u_{1}=\left[-\gamma \tanh ^{2}\left(\frac{\lambda^{2}-4 \mu}{2} \eta+\eta_{0}\right)+\gamma\right]^{\frac{1}{n}}
$$

while at $C_{1}^{2}<C_{2}^{2}$, one can obtain

$$
u_{1}=\left[-\gamma \operatorname{coth}^{2}\left(\frac{\lambda^{2}-4 \mu}{2} \eta+\eta_{0}\right)+\gamma\right]^{\frac{1}{n}}
$$

where $\eta_{0}=\tanh ^{-1}\left(\frac{C_{1}}{C_{2}}\right)$.

When $\lambda^{2}-4 \mu<0$,

$$
u_{2}=\left[\gamma\left(\frac{-C_{1} \sin \frac{1}{2} \sqrt{4 \mu-\lambda^{2}} \eta+C_{2} \cos \frac{1}{2} \sqrt{4 \mu-\lambda^{2}} \eta}{C_{1} \cos \frac{1}{2} \sqrt{4 \mu-\lambda^{2}} \eta+C_{2} \sin \frac{1}{2} \sqrt{4 \mu-\lambda^{2}} \eta}\right)^{2}+\gamma\right]^{\frac{1}{n}},
$$

where $\eta=k x \pm \frac{\sqrt{4 \beta \mu-\lambda^{2} \beta}}{n} t, \quad \gamma=\frac{\beta k^{2}\left(n^{2}+3 n+2\right)\left(\lambda^{2}-4 \mu\right)}{2 \alpha n^{2}}, C_{1}$ and $C_{2}$ are arbitrary constants. Similarity, it is easy to see that the trigonometric solution (16) can be rewritten at $C_{1}^{2}>C_{2}^{2}$ and $C_{1}^{2}<C_{2}^{2}$, as follows

$$
u_{2}=\left[\gamma \tan ^{2}\left(\frac{4 \mu-\lambda^{2}}{2} \eta+\eta_{0}\right)+\gamma\right]^{\frac{1}{n}}
$$

and

$$
u_{2}=\left[\gamma \cot ^{2}\left(\frac{4 \mu-\lambda^{2}}{2} \eta+\eta_{0}\right)+\gamma\right]^{\frac{1}{n}}
$$

where $\eta_{0}=\tan ^{-1}\left(\frac{C_{1}}{C_{2}}\right)$.

When $\lambda^{2}-4 \mu=0$,

$$
u_{3}=-\frac{2 \beta k^{2}\left(n^{2}+3 n+2\right)}{\alpha n^{2}} \frac{C_{2}^{2}}{\left(C_{1}+C_{2} \eta\right)^{2}},
$$

where $\eta=k x, C_{1}$ and $C_{2}$ are arbitrary constants.

Authors [29] have looked for exact solutions of Equation (3) using the wave variable $\xi=k(x-\lambda t)$, Equation (3) should become the ODE

$$
k^{2} \lambda^{2} U^{\prime \prime}+\alpha k^{2}\left(U^{n} U^{\prime}\right)^{\prime}+\beta k^{4} U^{\prime \prime \prime \prime}=0
$$

not the ODE (7) in [29] in the following form

$$
k^{2} \lambda^{2} U^{\prime \prime}+\alpha\left(k U^{n} U^{\prime}\right)^{\prime}+\beta k^{4} U^{\prime \prime \prime \prime}=0 .
$$

Therefore, the exact solutions given in [29] are wrong. To the best of our knowledge, solutions (13), (16) and (19) have not been reported in literature. 


\section{Conclusion}

The $\left(\frac{G^{\prime}}{G}\right)$-expansion method has been successfully applied here to seek exact solutions of the generalized Benjamin equation. As a result, a series of new exact solutions are obtained. The solution procedure is very simple and the travelling wave solutions are expressed by the hyperbolic functions, the trigonometric functions and the rational functions. To the best of our knowledge, these solutions have not been reported in literature. It is shown that the $\left(\frac{G^{\prime}}{G}\right)$-expansion method provides a very effective and powerful mathematical tool for solving nonlinear equations in mathematical physics.

\section{Acknowledgements}

This work was supported by the research project of Yuncheng University (No.YQ-2011013, YQ-2011068, XK2012004).

\section{References}

[1] Ablowitz, M.J. and Clarkson, P.A. (1991) Solitons, Nonlinear Evolution Equations and Inverse Scattering. Cambridge University Press, New York. http://dx.doi.org/10.1017/CBO9780511623998

[2] Hirota, R. (2004) The Direct Method in Soliton Theory. Cambridge University Press, New York. http://dx.doi.org/10.1017/CBO9780511543043

[3] Parkes, E.J. and Duffy, B.R. (1996) An Automated tanh-Function Method for Finding Solitary Wave Solutions to Nonlinear Evolution Equations. Computer Physics Communications, 98, 288-300. http://dx.doi.org/10.1016/0010-4655(96)00104-X

[4] Fan, E.G. (2000) Extended tanh-Function Method and Its Applications to Nonlinear Equations. Physics Letters A, 277, 212-218. http://dx.doi.org/10.1016/S0375-9601(00)00725-8

[5] Yan, C.T. (1996) A Simple Transformation for Nonlinear Waves. Physics Letters A, 224, 77-84. http://dx.doi.org/10.1016/S0375-9601(96)00770-0

[6] Wazwaz, A.M. (2003) A Study on Nonlinear Dispersive Partial Differential Equations of Compact and Noncompact Solutions. Applied Mathematics and Computation, 135, 399-409. http://dx.doi.org/10.1016/S0096-3003(02)00005-X

[7] Wang, M.L. (1995) Solitary Wave Solutions for Variant Boussinesq Equations. Physics Letters A, 199, 169-172. http://dx.doi.org/10.1016/0375-9601(95)00092-H

[8] Wang, M.L., Zhou, Y.B. and Li, Z.B. (1996) Applications of a Homogeneous Balance Method to Exact Solutions of Nonlinear Equations in Mathematical Physics. Physics Letters A, 216, 67-75. http://dx.doi.org/10.1016/0375-9601(96)00283-6

[9] Liu, S.K., Fu, Z.T. and Liu, S.D. (2001) Jacobi Elliptic Function Expansion Method and Periodic Wave Solutions of Nonlinear Wave Equations. Physics Letters A, 289, 69-74. http://dx.doi.org/10.1016/S0375-9601(01)00580-1

[10] Fu, Z.T., Liu, S.K. and Liu, S.D. (2001) New Jacobi Elliptic Function Expansion and New Periodic Wave Solutions of Nonlinear Wave Equations. Physics Letters A, 290, 72-76. http://dx.doi.org/10.1016/S0375-9601(01)00644-2

[11] Feng, Z.S. (2002) On Explicit Exact Solutions to the Compound Burgers-KdV Equation. Physics Letters A, 293, 57-66. http://dx.doi.org/10.1016/S0375-9601(01)00825-8

[12] Feng, Z.S. (2002) Exact Solution to an Approximate Sine-Gordon Equation in (n + 1)-Dimensional Space. Physics Letters A, 302, 64-76. http://dx.doi.org/10.1016/S0375-9601(02)01114-3

[13] Chen, Y. and Yan, Z.Y. (2005) New Exact Solutions of $(2+1)$-Dimensional Gardner Equation via the New Sine-Gordon Equation Expansion Method. Chaos, Solitons \& Fractals, 26, 399-406. http://dx.doi.org/10.1016/j.chaos.2005.01.004

[14] He, J.H. and Wu, X.H. (2006) Exp-Function Method for Nonlinear Wave Equations. Chaos, Solitons \& Fractals, 30, 700-708. http://dx.doi.org/10.1016/j.chaos.2006.03.020

[15] He, J.H. and Abdou, M.A. (2007) New Periodic Solutions for Nonlinear Evolutions Using Exp-Function Method, Chaos, Solitons \& Fractals, 34, 1421-1429. http://dx.doi.org/10.1016/j.chaos.2006.05.072

[16] Gao, H. and Zhao, R.X. (2010) New Exact Solutions to the Generalized Burgers-Huxley Equation. Applied Mathematics and Computation, 217, 1598-1603. http://dx.doi.org/10.1016/j.amc.2009.07.020

[17] Wang, M.L., Li, X.Z. and Zhang, J.L. (2008) The $\left(\frac{G^{\prime}}{G}\right)$-Expansion Method and Travelling Wave Solutions of Nonli- 
near Evolution Equations in Mathematical Physics. Physics Letters A, 372, 417-423. http://dx.doi.org/10.1016/j.physleta.2007.07.051

[18] Bekir, A. (2008) Application of the $\left(\frac{G^{\prime}}{G}\right)$-Expansion Method for Nonlinear Evolution Equations. Physics Letters A, 372, 3400-3406. http://dx.doi.org/10.1016/j.physleta.2008.01.057

[19] Wang, M.L., Zhang, J.L. and Li, X.Z. (2008) Application of the ( $\left.\frac{G^{\prime}}{G}\right)$-Expansion to Travelling Wave Solutions of the Broer-Kaup and the Approximate Long Water Wave Equations. Applied Mathematics and Computation, 206, 321-326. http://dx.doi.org/10.1016/j.amc.2008.08.045

[20] Zhang, J., Wei, X. and Lu, Y. (2008) A generalized $\left(\frac{G^{\prime}}{G}\right)$-Expansion Method and Its Applications. Physics Letters A, 372, 3653-3658. http://dx.doi.org/10.1016/j.physleta.2008.02.027

[21] Zhang, S., Tong, J.L. and Wang, W. (2008) A Generalized $\left(\frac{G^{\prime}}{G}\right)$-Expansion Method for the mKdV Equation with Variable Coefficients. Physics Letters A, 372, 2254-2257. http://dx.doi.org/10.1016/j.physleta.2007.11.026

[22] Zhang, S., Dong, L., Ba, J.M. and Sun, Y.N. (2009) The $\left(\frac{G^{\prime}}{G}\right)$-Expansion Method for Nonlinear Differential-Difference Equations. Physics Letters A, 373, 905-910. http://dx.doi.org/10.1016/j.physleta.2009.01.018

[23] Zhang, H.Q. (2009) New Application of the $\left(\frac{G^{\prime}}{G}\right)$-Expansion Method. Communications in Nonlinear Science and Numerical Simulation, 14, 3220-3225. http://dx.doi.org/10.1016/j.cnsns.2009.01.006

[24] Aslan, İ. and Öziş, T. (2009) Analytic Study on Two Nonlinear Evolution Equations by Using the (G'/G)-Expansion Method. Applied Mathematics and Computation, 209, 425-429. http://dx.doi.org/10.1016/j.amc.2008.12.064

[25] Öziş, T. and Aslan, İ. (2009) Symbolic Computation and Construction of New Exact Traveling Wave Solutions to Fitzhugh-Nagumo and Klein-Gordon Equations. Zeitschrift für Naturforschung, 64a, 15-20.

[26] Öziş, T. and Aslan, İ. (2009) Symbolic Computations and Exact and Explicit Solutions of Some Nonlinear Evolution Equations in Mathematical Physics. Communications in Theoretical Physics, 51, 577-580. http://dx.doi.org/10.1088/0253-6102/51/4/01

[27] Gao, H. and Zhao, R.X. (2009) New Application of the (G'/G)-Expansion Method to Higher-Order Nonliear Equations. Applied Mathematics and Computation, 215, 2781-2786. http://dx.doi.org/10.1016/j.amc.2009.08.041

[28] Hereman, W., Banerjee, P.P. and Korpel, A. (1986) Exact Solitary Wave Solutions of Nonliear Evolution and Wave Equations Using a Direct Algebraic Method. Journal of Physics A: Mathematical and General, 19, 607-628. http://dx.doi.org/10.1088/0305-4470/19/5/016

[29] Taghizadeh, N., Mirzazadeh, M. and Moosavi Noori, S.R. (2012) Exact Solutions of the Generalized Benjamin Equation and (3 + 1)-Dimensional Gkp Equation by the Extended tanh Method. Applications \& Applied Mathematics, 7, 175-187. 
Scientific Research Publishing (SCIRP) is one of the largest Open Access journal publishers. It is currently publishing more than 200 open access, online, peer-reviewed journals covering a wide range of academic disciplines. SCIRP serves the worldwide academic communities and contributes to the progress and application of science with its publication.

Other selected journals from SCIRP are listed as below. Submit your manuscript to us via either submit@scirp.org or Online Submission Portal.
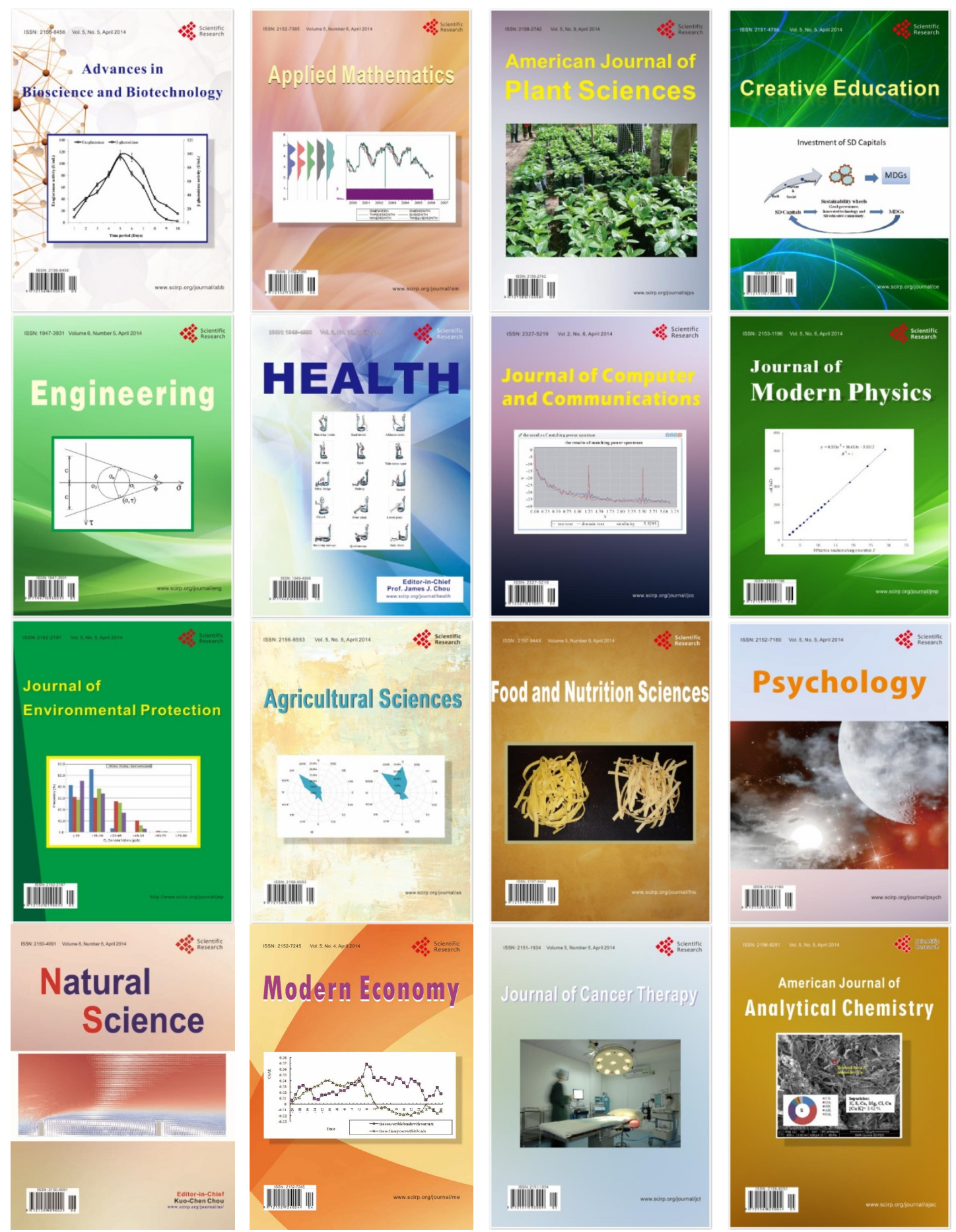